\title{
ON A GENERALISED CESÀRO SUMMABILITY METHOD OF INTEGRAL ORDER
}

D. BORWEIN

(Received October 11. 1965)

Let $p$ be a non-negative integer, $\left\{\lambda_{n}\right\}$ a strictly increasing unbounded sequence with $\lambda_{0} \geqq 0$, and let $\sum_{n=0}^{\infty} a_{n}$ be an arbitrary series. Write

$$
\begin{gathered}
A^{p}(w)=\sum_{\lambda_{n}<w}\left(w-\lambda_{n}\right)^{p} a_{n} \quad(w \geqq 0) ; \\
C_{n}^{0}=\sum_{\nu=0}^{n} a_{\nu}, C_{n}^{p}=\sum_{\nu=0}^{n}\left(\lambda_{n+1}-\lambda_{\nu}\right) \cdots\left(\lambda_{n+p}-\lambda_{\nu}\right) a_{\nu} \quad(p \geqq 1) ; \\
\lambda_{n, 0}=1, \lambda_{n, p}=\lambda_{n+1} \cdots \lambda_{n+p} \quad(p \geqq 1) .
\end{gathered}
$$

The series $\sum a_{n}$ is said to be

(i) summable by the Riesz method $(R, \lambda, p)$ to $s$ if $w^{-p} A^{p}(w) \rightarrow s$ as $w \rightarrow \infty$,

(ii) summable by the generalised Cesàro method $(C, \lambda, p)$ to $s$ if $C_{n}^{p} / \lambda_{n, p} \rightarrow s$ as $n \rightarrow \infty$.

The relationship between these two summability methods has been investigated by Jurkat [2] and Burkill [1], who independently defined generalised Cesàro methods essentially the same as the above; and by Russell [3]. All three established the inclusions

$$
\begin{aligned}
& \left(\mathrm{I}_{1}\right): \quad(C, \lambda, p) \subseteq(R, \lambda, p), \\
& \left(\mathrm{I}_{2}\right): \quad(R, \lambda, p) \subseteq(C, \lambda, p)
\end{aligned}
$$

under various hypotheses on the sequence $\left\{\lambda_{n}\right\}$. The most general results to date are due to Russell [3], who proved that $\left(\mathrm{I}_{1}\right)$ holds without restriction on $\left\{\lambda_{n}\right\}$, and that $\left(\mathrm{I}_{2}\right)$ holds provided

$$
\left(C_{1}\right): \frac{\lambda_{n}}{\lambda_{n}-\lambda_{n-1}}=O\left(\frac{\lambda_{n+1}}{\lambda_{n+1}-\lambda_{n}}\right)
$$


when $p \geqq 3$ and unrestrictedly when $p \leqq 2$.

The object of this note is to prove that $\left(I_{2}\right)$ also holds if

$$
\left(C_{2}\right): \lambda_{n+1}=O\left(\lambda_{n}\right)
$$

Conditions $\left(C_{1}\right)$ and $\left(C_{2}\right)$ are independent (see [3]).

We shall prove the following theorem in which there is no restriction on $\left\{\lambda_{n}\right\}$.

THEOREM. If $\eta(w)$ is non-negative and monotonic non-decreasing for $w \geqq 0$ and $A^{p}(w)=o(\eta(w))$ as $w \rightarrow \infty$, then $C_{n}^{p}=o\left(\eta\left(\lambda_{n+p}\right)\right)$.

The theorem remains valid if $o$ is replaced by $O$ throughout.

As an immediate corollary we have:

COROLlaRY. If $\left(C_{2}\right)$ and $A^{p}(w)=o\left(w^{p}\right)$ as $w \rightarrow \infty$, then $C_{n}^{p}=o\left(\lambda_{n+1}^{p}\right)$ $=o\left(\lambda_{n, p}\right)$.

Since the methods $(C, \lambda, p)$ and $(R, \lambda, p)$ are both regular (see [3], Cor. 1B), a consequence of the corollary is that $\left(C_{2}\right)$ is a sufficient condition for inclusion $\left(\mathrm{I}_{2}\right)$.

PROOF OF THE THEOREM. Since the theorem is trivially true when $p=0$, we shall assume that $p \geqq 1$. Let $m=m(n)$ be an integer such that

$$
\lambda_{m+1}-\lambda_{m}=\max _{n \leqq i \leqq n+p-1}\left(\lambda_{i+1}-\lambda_{i}\right), \quad n \leqq m \leqq n+p-1,
$$

and let

$$
b_{i}=b_{n, i}=(p+1) \frac{\lambda_{n+i}-\lambda_{m}}{\lambda_{m+1}-\lambda_{m}} \quad(i=1,2, \cdots, p) .
$$

Then (c.f. Burkill [1], p. 57) there are numbers $y_{i}=y_{n, i}(i=0,1, \cdots, p)$ such that

$$
\begin{array}{r}
\left(x+b_{1}\right)\left(x+b_{2}\right) \cdots\left(x+b_{p}\right) \equiv y_{0}(x+1)^{p}+y_{1}(x+2)^{p} \\
+\cdots+y_{p}(x+p+1)^{p},
\end{array}
$$

which is equivalent to the system of linear equations

$$
\sum_{j=0}^{p}(j+1)^{i} y_{i}=k_{i} \quad(i=0,1, \cdots, p)
$$

where 


$$
k_{i}=\left(\begin{array}{c}
p \\
i
\end{array}\right)^{-1} \sum_{1 \leqq r_{1}<\cdots<r_{i} \leqq p} b_{r_{1}} b_{r_{2}} \cdots b_{r_{i}} .
$$

The determinant of the system (2) is

$$
\Delta=\prod_{1 \leqq r<s \leqq p+1}(s-r) \geqq 1
$$

and $y_{r}=\Delta_{r} / \Delta$ where $\Delta_{r}$ is the determinant of the matrix

$$
\left(c_{i, j}\right)(i, j=0,1, \cdots, p) \text { where } c_{i, r}=k_{i} \text { and } c_{i, j}=(j+1)^{i}(j \neq r) \text {. }
$$

Since $\left|b_{r}\right|<(p+1)^{2}$, we see that $\left|k_{i}\right|<(p+1)^{2 p}$ and hence that $\left|c_{i, j}\right|<(p+1)^{2 p}$. Consequently

$$
\left|y_{r}\right|=\left|y_{n, r}\right| \leqq\left|\Delta_{r}\right|<(p+1) !(p+1)^{2 p} \quad(r=0,1, \cdots, p ; n=0,1, \cdots) .
$$

Putting $\quad x=(p+1) \frac{\lambda_{m}-\lambda_{\nu}}{\lambda_{m+1}-\lambda_{m}}$ in identity (1), we obtain

$$
\left(\lambda_{n+1}-\lambda_{\nu}\right) \cdots\left(\lambda_{n+p}-\lambda_{\nu}\right)=y_{n, 0}\left(\mu_{n, 0}-\lambda_{\nu}\right)^{p}+\cdots+y_{n, p}\left(\mu_{n, p}-\lambda_{\nu}\right)^{p}
$$

where

$$
\begin{aligned}
\lambda_{m}<\mu_{n, i}=\lambda_{m}+\frac{i+1}{p+1}\left(\lambda_{m+1}-\lambda_{m}\right) \leqq \lambda_{m+1} \leqq \lambda_{n+p} \\
(i=0,1, \cdots, p ; n=0,1, \cdots) .
\end{aligned}
$$

Hence

$$
C_{n}^{p}=\sum_{\nu=0}^{m}\left(\lambda_{n+1}-\lambda_{\nu}\right) \cdots\left(\lambda_{n+p}-\lambda_{\nu}\right) a_{\nu}=\sum_{i=0}^{p} y_{n, i} A^{p}\left(\mu_{n, i}\right) ;
$$

and the theorem is an immediate consequence of (3), (4) and (5).

\section{REFERENCES}

[1] H. Burkill., On Riesz and Riemann Summability, Proc. Camb. Phil. Soc., 57(1961), $55-60$.

[2] W. B. JURKAT, Über Rieszsche Mittel und verwandte Klassen von Matrixtransformationen, Math. Zeit., 57(1953), 353-394.

[3] D. C. Russell, On generalised Cesàro means of integral order, Tôhoku Math. Journ., 17 (1965), 410-442.

\section{UNIVERSITY OF WESTERN ONTARIO}

LONDON, CANADA. 